\title{
Solar wind driven dust acoustic instability with Lorentzian kappa distribution
}

\author{
Kashif Arshad, ${ }^{1,2}$ Zahida Ehsan, ${ }^{1,3,4, a)}$ S. A. Khan, ${ }^{1}$ and S. Mahmood ${ }^{5}$ \\ ${ }^{1}$ National Center for Physics (NCP), Quaid-i-Azam University Campus, Shahdra Valley Road, \\ Islamabad 44000, Pakistan \\ ${ }^{2}$ Pakistan Institute of Engineering and Applied Sciences, P.O. Nilore, Islamabad and University of Wah, \\ Wah Cantt 47040, Pakistan \\ ${ }^{3}$ Universita degli Studi del Molise, 86090 Pesche (IS), Italy and INFN Sezione di Napoli, 80126 Napoli, Italy \\ ${ }^{4}$ Department of Physics, COMSATS Institute of Information Technology (CIIT), Defence Road, \\ Off Raiwind Road, Lahore 86090, Pakistan \\ ${ }^{5}$ Theoretical Plasma Physics Division, PINSTEC, PO Box Nilore, Islamabad 44000, Pakistan
}

(Received 8 October 2013; accepted 30 January 2014; published online 18 February 2014)

In a three species electron-ion-dust plasma following a generalized non-Maxwellian distribution function (Lorentzian or kappa), it is shown that a kinetic instability of dust-acoustic mode exists. The instability threshold is affected when such (quasineutral) plasma permeates through another static plasma. Such case is of interest when the solar wind is streaming through the cometary plasma in the presence of interstellar dust. In the limits of phase velocity of the waves larger and smaller than the thermal velocity of dust particles, the dispersion properties and growth rate of dust-acoustic mode are investigated analytically with validation via numerical analysis. (c) 2014 AIP Publishing LLC. [http://dx.doi.org/10.1063/1.4865573]

\section{INTRODUCTION}

When two or more plasmas encounter, an interpenetration of species takes place and one plasma species can permeate the other. This is the so-called permeating plasma. ${ }^{1-5}$ The permeating plasma can be modeled considering two parts: the flowing plasma and the target (may or may not be static). The flowing plasma has the dynamics on faster time scale than the target plasma which is in a relative static state, and can permeate through it. Such situation can be created in laboratory conditions, while in space this happens quite frequently, for instance, when astrophysical clouds collide, when plasmas originating from the explosions of novae and supernovae propagate and move through the surrounding plasmas. ${ }^{1}$ Another example of such a permeating plasma is the inter-penetrating solar wind and surrounding cometary plasma. Commonly, the solar/stellar wind is dominated by electron ion plasma, while the cometary plasma is a dusty plasma which consists of charged and heavy particles other than electrons and ions, ${ }^{5-7}$ and sometimes even both positive and negative ions. ${ }^{8}$ In the vicinity of Earth, a longer period dust showers from comets encounter the dusty plasmas in the earth's atmosphere and a situation of permeating dusty plasma happens. Such type of interactions of two plasmas in the interplanetary space is frequent which, in turn makes interesting to investigate of the basic properties of dusty plasma under such environment, waves, instabilities, structure formation, and so on. ${ }^{5}$

In a recent study of permeating plasma dynamics, a kinetic current-less instability was found for two interpenetrating plasmas where the threshold velocity of the propagating plasma was even below the ion-acoustic (IA) speed of static plasma. ${ }^{1}$ In such (frequent) configurations in cometary magnetospheres, and interpenetrating space and astrophysical

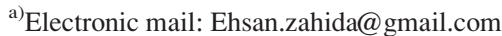

plasmas, this type of instability was found much more effective than in ordinary plasmas. ${ }^{2}$

It is important to point out that for being currentless, it also eliminates the difficulties associated with the selfinduced magnetic field associated with the current due to background electrons. ${ }^{1}$ The investigation of such kind of kinetic instability was carried out in the framework of a thermodynamically stable plasma obeying the Maxwellian behavior. However in many realistic situations of space plasmas, the Maxwellian distribution is not an appropriate choice since plasmas are more likely to incur various effects, e.g., the presence of energetic electrons, spatial changes in plasma properties, external force fields, etc., which can complicate the flows of particles. For these obvious reasons, the natural space plasmas have been frequently observed to have nonMaxwellian behavior with high-energy tails or have flat tops with pronounced shoulders. The deviation of plasmas from Maxwellian behavior is also been noted in the laboratory. ${ }^{9}$ At low energies, the distribution appears reasonably Maxwellian but have "superthermal tail" at high energies.

The plasma distribution with superthermality effects is well modelled by a generalized distribution function-the Lorentzian or kappa distribution function. ${ }^{10-12}$ Recently, study of different phenomena in plasmas with kappa distribution has received a great deal of attraction for its many interesting applications found in the wide fields of space plasma physics and astrophysics. ${ }^{12-16}$ For example, nonMaxwellian distribution function has been used to study ion (dust) acoustic waves, ${ }^{14,15}$ solitons, ${ }^{16}$ the solar interior, ${ }^{17}$ the solar wind, and the interplanetary space plasma, ${ }^{18,19}$ etc.

Permeating dusty plasma being a complex plasma system is far away from the equilibrium. Thus, the plasma particles will not follow a Maxwellian distribution and, in many physical situations, will asymptotically approach to a nonequilibrium stationary-state with power-law distributions, e.g., often fitted with the kappa-distribution. A generalized description of 
a three-dimensional kappa distribution function is given by $^{20-27}$

$$
f_{\kappa}=\frac{n_{\alpha_{0}}}{\pi^{\frac{3}{2}} \theta_{\perp_{\alpha}}^{2} \theta_{\|_{\alpha}}} \frac{\Gamma(\kappa+1)}{\kappa^{\frac{3}{2}} \Gamma\left(\kappa-\frac{1}{2}\right)}\left[1+\frac{v_{\|}^{2}}{\kappa \theta_{\|_{\alpha}}^{2}}+\frac{v_{\perp}^{2}}{\kappa \theta_{\perp_{\alpha}}^{2}}\right]^{-\kappa-1},
$$

where $n_{\alpha 0}$ is the unperturbed plasma number density and $\kappa$ is the spectral index that determines the hardness of the energy spectrum corresponding to the suprathermal tail of the distribution function. The thermal speed is related to the particle temperature $T$ in terms of the parameter $\theta$ as $\theta_{(\|, \perp)_{\alpha}}=[(2 \kappa-3) / \kappa]^{1 / 2} v_{T_{(\|, \perp)_{\alpha}}}$ with $v_{T_{(\|, \perp)_{\alpha}}}=\left[\left(T_{\|, \perp}\right)_{\alpha} / m_{\alpha}\right]^{1 / 2}$ which is defined only for $\kappa>3 / 2$. Furthermore, $f_{\kappa}$ is normalized to unity, i.e., $\int f_{\kappa} d^{3} v=1$, and $\Gamma$ is the mathematical (gamma) function. The function (1) leads to Maxwellian behavior for $\kappa \rightarrow \infty$, whereas it shows a strong non-Maxwellian tail obeying a power-law for low values of $\kappa$. This function has been widely used in the general physics context and the kinetic and fluid modeling of the dynamics of plasmas, e.g., see Refs. 12-16 and 18 .

In this paper, we study the low frequency dynamics of an electron-ion-dust plasma obeying the distribution function (1). We investigate the dispersion properties and existence of a kinetic instability of dust-acoustic wave when a dusty plasma permeates through another static plasma, a situation relevant to the interstellar plasmas. The paper is organized as follows. In Sec. II, we present the basic (linearized) formulation for the dynamics of dust grains (dust-acoustic waves) via the dielectric function and obtain the dispersion equation and the instability conditions. Section III is devoted to the quantitative analysis and conclusions on the dust-acoustic wave instability driven by a cometary plasma trail passing through the interplanetary space.

\section{DISPERSION AND GROWTH OF WAVES IN NON-MAXWELLIAN PLASMAS}

Using the kinetic theory, we will investigate the instability of dust acoustic waves driven by the solar wind plasma.

Let us consider a multispecies collisionless magneticfield-free plasma comprising electrons, ions, and negatively charged dust particles following the distribution function (1). ${ }^{8-10}$

In the space dusty plasmas, although the electrons are more likely to follow the non-Maxwellian distribution than the ions and the dust particles, yet it seems quite reasonable that, as a general discussion, the plasma components are given a unified form of the kappa distribution ${ }^{17}$ In addition, observations of the solar winds have shown the presence of the high-velocity tails on the velocity distributions of the ions. The tails are usually made up of particles accelerated from the ambient solar wind by the shocks. Compared with the electrons and ions, the dust grains might have enough time (due to inertia) to be thermalized and thus might not deviate far from the Maxwellian distribution. However, for the mathematical convenience, we assume all species to behave kappa distribution and derive the expressions for the growth rate and later at the time of quantitative analysis, we use very small value of dust thermal velocity. The dielectric response function for such a plasma by employing the linearized Vlasov equation can be written as ${ }^{16,17}$

$$
\epsilon(k, \omega)=1-\Sigma_{\alpha} \frac{2}{\omega_{p_{\alpha}} k^{2} \lambda_{D_{\alpha}}^{3}} \frac{\Gamma(\kappa+1)}{(2 \kappa-3)^{\frac{3}{2}} \Gamma\left(\kappa-\frac{1}{2}\right)} Z\left(\frac{\omega}{k \theta_{\| \alpha}}\right),
$$

where $\omega_{p_{\alpha}}=\sqrt{4 \pi n_{\alpha 0} e^{2} / m_{\alpha}}$ is the plasma frequency and $\lambda_{D_{\alpha}}=\sqrt{T_{\alpha} / 4 \pi n_{\alpha 0} e^{2}}$ is the Debye length of the $\alpha$ species which represents the electron, ion, and dust, respectively, which will be elaborated for background and permeating species in a while. The integration is performed over the perpendicular velocity component and the plasma dispersion function in this case is defined as

$$
Z\left(\frac{\omega}{k \theta_{\| \alpha}}\right)=\frac{1}{\sqrt{\pi}} \int_{-\infty}^{\infty}\left(1+\frac{v_{\|}^{2}}{\kappa \theta_{\| \alpha}^{2}}\right)^{-\kappa-1} \frac{v_{\|}}{\left(v_{\|}-v_{\phi}\right)} d \mathbf{v}_{\|}
$$

with $v_{\phi}=|\omega / k|$ being the phase velocity. In the limit of small $\left(v_{\phi} \ll \theta_{\| \alpha}\right)$ and large $\left(v_{\phi} \gg \theta_{\| \alpha}\right)$ phase velocity, we obtain

$$
\begin{aligned}
Z\left(\frac{\omega}{k \theta_{\| \alpha}}\right)= & \left(\theta_{\| \alpha} \kappa^{1 / 2} \frac{\Gamma(\kappa+1 / 2)}{\Gamma(\kappa+1)}\right) \\
& +i \sqrt{\pi}\left(\frac{\omega}{k}\right)\left(1+\frac{\omega^{2}}{\kappa k^{2} \theta_{\| \alpha}^{2}}\right)^{-\kappa-1},
\end{aligned}
$$

and

$$
\begin{aligned}
Z\left(\frac{\omega}{k \theta_{\| \alpha}}\right)= & \theta_{\| \alpha} \kappa^{3 / 2} \frac{\Gamma(\kappa-1 / 2)}{\Gamma(\kappa+1)}\left(\frac{k \theta_{\| \alpha}}{\omega}\right)^{2} \\
& \times\left[1+\frac{3 \kappa}{2(3 \kappa-2)}\left(\frac{k \theta_{\| \alpha}}{\omega}\right)^{2}\right] \\
& +\ldots \ldots \ldots \ldots-i \sqrt{\pi}\left(\frac{\omega}{k}\right)\left[1+\frac{\omega^{2}}{\kappa k^{2} \theta_{\| \alpha}^{2}}\right]^{-\kappa-1}
\end{aligned}
$$

respectively. Now, we consider our permeating plasma comprises a flowing (e.g., solar wind) and relatively stationary or target plasma (e.g., cometary dusty plasma). Let the background number densities are $n_{0_{\omega j}}$ (solar wind plasma), $n_{0_{c j}}$ (cometary plasma), and $n_{0_{d}}$ (static dust grains), where the index $j=e$ and $i$ stand for electrons and ion species. The charge neutrality condition of such a permeating plasma is given by

$$
n_{0_{c e}}+n_{0_{\omega e}}=n_{0_{c i}}+n_{0_{\omega i}}+\eta Z_{d} n_{0_{d}}
$$

where $\eta$ represents nature of the dust charge (positive or negative) and $Z_{d}$ is the charging parameter of dust grains.

To model the dispersion relations and growth rates in ultra-low (dust) frequency modes, we select the appropriate limits of phase velocity for all the plasma components. The dust grains are treated in large phase velocity limit, $v_{\phi} \gg \theta_{\|_{d}}$ due to their large mass/inertia and the phase velocity limit of cometary plasma is assumed $v_{\phi} \gg \theta_{\|_{c e}}, \theta_{\|_{c i}}$. However, the solar wind can act as a source of free energy to play a dominant role in the growth of the instability and/or wave excitation due to very large plasma streaming velocity $\sim \times 10^{7} \mathrm{~cm} / \mathrm{s}$. 
Obviously, the inertia of wind electrons is ignorable in the limit $v_{\phi} \gg \theta_{\|_{\omega e}}$, while the inertia of wind ions is crucial in a ultra-low frequency mode. To check the role of wind ions, at first they are treated in the large phase velocity limit $v_{\phi} \gg \theta_{\|_{\omega i}}$ for the calculation of the growth rate. Then, we will disregard their inertia in the limit $v_{\phi} \ll \theta_{\|_{\omega i}}$. Hence, under the large $v_{\phi}$ limit of the ions of the solar wind plasma, one can write the dielectric response function as follows:

$$
\begin{aligned}
& \epsilon(k, \omega)=\left(2 \frac{\left(\kappa-\frac{1}{2}\right)}{\left(\kappa-\frac{3}{2}\right)}+k^{2} \lambda_{D_{2}}^{2}\right)-\frac{k^{2} \lambda_{D_{2}}^{2} \omega_{p_{\omega i}}^{2}}{\left(\omega-k v_{0}\right)^{2}} \times\left(1+\frac{k^{2} \theta_{\|_{\omega i}}^{2}}{\left(\omega-k v_{0}\right)^{2}} \frac{3 \kappa}{2\left(\kappa-\frac{3}{2}\right)}\right)-\frac{k^{2} \lambda_{D_{2}}^{2} \omega_{p_{d}}^{2}}{\omega^{2}}\left(1+\frac{k^{2} \theta_{\|_{d}}^{2}}{\omega^{2}} \frac{3 \kappa}{2\left(\kappa-\frac{3}{2}\right)}\right) \\
& +2 i \sqrt{\pi} \frac{\lambda_{D_{2}}^{2} \Gamma(\kappa+1)}{\kappa^{\frac{3}{2}} \Gamma\left(\kappa-\frac{1}{2}\right)}\left[\frac{\omega / k}{\omega_{p_{c i}} \lambda_{D_{c i}}^{3}}+\frac{\omega / k}{\omega_{p_{c e}} \lambda_{D_{c e}}^{3}}+\frac{\omega / k-v_{0}}{\omega_{p_{w e}} \lambda_{D_{w e}}^{3}}+\frac{\omega / k-v_{0}}{\omega_{p_{w i}} \lambda_{D_{w i}}^{3}}\left(1+\frac{\left(\omega / k-v_{0}\right)^{2}}{\kappa \theta_{\|_{\omega i}}^{2}}\right)^{-\kappa-1}\right. \\
& \left.+\frac{\omega / k}{\omega_{p_{d}} \lambda_{D_{d}}^{3}}\left(1+\frac{\left(\frac{\omega}{k}\right)^{2}}{\kappa \theta_{\|_{d}}^{2}}\right)^{-\kappa-1}\right]
\end{aligned}
$$

where $\lambda_{D_{2}}^{-2}=\lambda_{D_{c i}}^{-2}+\lambda_{D_{\omega e}}^{-2}+\lambda_{D_{c e}}^{-2}$ is the effective Debye length. The above dielectric function is composed of real part $\epsilon_{r}$ and an imaginary part $\epsilon_{i}$ such that $\epsilon(k, \omega)=\epsilon_{r}+i \epsilon_{i}$. We obtain the dispersion relation of the respective mode by equating the respective relation $\left(\epsilon_{r}\right.$ or $\left.\epsilon_{i}\right)$ to zero. Then, for real $\omega$, we obtain

$$
\omega_{r}^{2}=\frac{k^{2} \lambda_{D_{2}}^{2} \omega_{p_{d}}^{2}}{\left(2 \frac{\left(\kappa-\frac{1}{2}\right)}{\left(\kappa-\frac{3}{2}\right)}+k^{2} \lambda_{D_{2}}^{2}\right)}\left[\left(1+\frac{k^{2} \theta_{\|_{d}}^{2}}{\omega^{2}} \frac{3 \kappa}{(2 \kappa-3)}\right)-\frac{\omega^{2} \omega_{p_{\omega i}}^{2}}{\omega_{p_{d}}^{2}\left(\omega-k v_{0}\right)^{2}}\left(1+\frac{k^{2} \theta_{\|_{w i}}^{2}}{\left(\omega-k v_{0}\right)^{2}} \frac{3 \kappa}{(2 \kappa-3)}\right)\right] .
$$

As $k v_{T} /\left(\omega-k v_{0}\right) \ll 1$, we can write the above relation as

$$
\omega_{r}^{2}=\frac{T_{c e} / m_{d}}{\left(1+\frac{T_{c e}}{T_{c i}} \frac{n_{c i 0}}{n_{c e 0}}+\frac{T_{c e}}{T_{w e}} \frac{n_{w e 0}}{n_{c e 0}}\right)} \frac{k^{2} Z_{d}^{2} n_{d o} / n_{c e 0}}{\left(\kappa \frac{\left(\kappa-\frac{1}{2}\right)}{\left(\kappa-\frac{3}{2}\right)}+k^{2} \lambda_{D_{2}}^{2}\right)}\left[1+\frac{k^{2} \theta_{\|_{d}}^{2}}{\omega^{2}} \frac{3 \kappa}{2\left(\kappa-\frac{3}{2}\right)}\right] .
$$

Finally, the imaginary part (growth) can be calculated from the well known formula $\gamma=-\epsilon_{i} /\left(\partial \epsilon_{r} / \partial \omega_{r}\right)$. After some algebra, we obtain the growth rate

$$
\begin{aligned}
& \gamma=\sqrt{\frac{\pi}{8}} \frac{\Gamma(\kappa+1)}{\left(\kappa-\frac{3}{2}\right)^{3 / 2} \Gamma\left(\kappa-\frac{1}{2}\right)} \frac{\omega_{p_{d}} \lambda_{D_{2}}^{3}}{\left(2 \frac{\left(\kappa-\frac{1}{2}\right)}{\left(\kappa-\frac{3}{2}\right)}+k^{2} \lambda_{D_{2}}^{2}\right)^{3 / 2}} \times\left\{v _ { 0 } \left(\frac{1}{\omega_{p_{w e}} \lambda_{D_{w e}}^{3}}+\frac{1}{\omega_{p_{w i}} \lambda_{D_{w i}}^{3}}\left[1+\frac{\omega^{2}\left(1-\frac{k v_{0}}{\omega}\right)^{2}}{\kappa k^{2} \theta_{\|_{w i}}^{2}}\right]^{-\kappa-1}\right.\right. \\
& \left.-\frac{\omega_{r}}{k}\left[\frac{1}{\omega_{p_{c i}} \lambda_{D_{c i}}^{3}}+\frac{1}{\omega_{p_{c e}} \lambda_{D_{c e}}^{3}}+\frac{1}{\omega_{p_{w e}} \lambda_{D_{w e}}^{3}}+\frac{1}{\omega_{p_{w i}} \lambda_{D_{w i}}^{3}}\left[1+\frac{\omega^{2}\left(1-\frac{k v_{0}}{\omega}\right)^{2}}{\kappa k^{2} \theta_{\|_{w i}}^{2}}\right]^{-\kappa-1}+\frac{1}{\omega_{p_{d}} \lambda_{D_{d}}^{3}}\left[1+\frac{\omega^{2}}{\kappa k^{2} \theta_{\|_{d}}^{2}}\right]^{-\kappa-1}\right]\right\}
\end{aligned}
$$


The above equation can be written in another form as

$$
\gamma=\left[v_{0} d-\frac{\omega_{r}}{k}(a+d+c)\right],
$$

where

$$
\begin{aligned}
& X=1+\left[1-\frac{\lambda_{D_{d}} v_{0}}{\lambda_{D_{2}} v_{T_{\|_{d}}}} \sqrt{2 \frac{\left(\kappa-\frac{1}{2}\right)}{\left(\kappa-\frac{3}{2}\right)}+k^{2} \lambda_{D_{2}}^{2}}\right]^{2} \times\left[\frac{\lambda_{D_{2}}^{2} v_{T_{\|_{d}}}^{2} / \lambda_{D_{d}}^{2} v_{T_{\|_{w i}}}^{2}}{(2 \kappa-3)\left(\frac{\left(\kappa-\frac{1}{2}\right)}{2}+k^{2} \lambda_{D_{2}}^{2}\right)}+\frac{3 v_{T_{\|_{d}}}^{2} / v_{T_{\|_{w i}}}^{2}}{(2 \kappa-3)}\right]^{-\kappa-1}, \\
& a=\frac{1}{\omega_{p_{c i}} \lambda_{D_{c i}}^{3}}+\frac{1}{\omega_{p_{c e}} \lambda_{D_{c e}}^{3}}, \\
& d=\frac{1}{\omega_{p_{w e}} \lambda_{D_{w e}}^{3}}+\frac{X}{\omega_{p_{w i}} \lambda_{D_{w i}}^{3}},
\end{aligned}
$$

and

$$
c=\frac{1}{\omega_{p_{d}} \lambda_{D_{d}}^{3}}\left[1+\frac{1 /(2 \kappa-3)}{\left(2 \frac{\left(\kappa-\frac{1}{2}\right)}{\left(\kappa-\frac{3}{2}\right)}+k^{2} \lambda_{D_{2}}^{2}\right)} \frac{\lambda_{D_{2}}^{2}}{\lambda_{D_{d}}^{2}}+\frac{3}{(2 \kappa-3)} \frac{\lambda_{D_{d}}^{2}}{\lambda_{D_{2}}^{2}}\right]^{-\kappa-1} .
$$

Hence the instability occurs for

$$
v_{0}>\frac{\omega_{r}}{k}\left[1+\left(\frac{a+c}{d}\right)\right] \text {, }
$$

and the growth rate can be written as
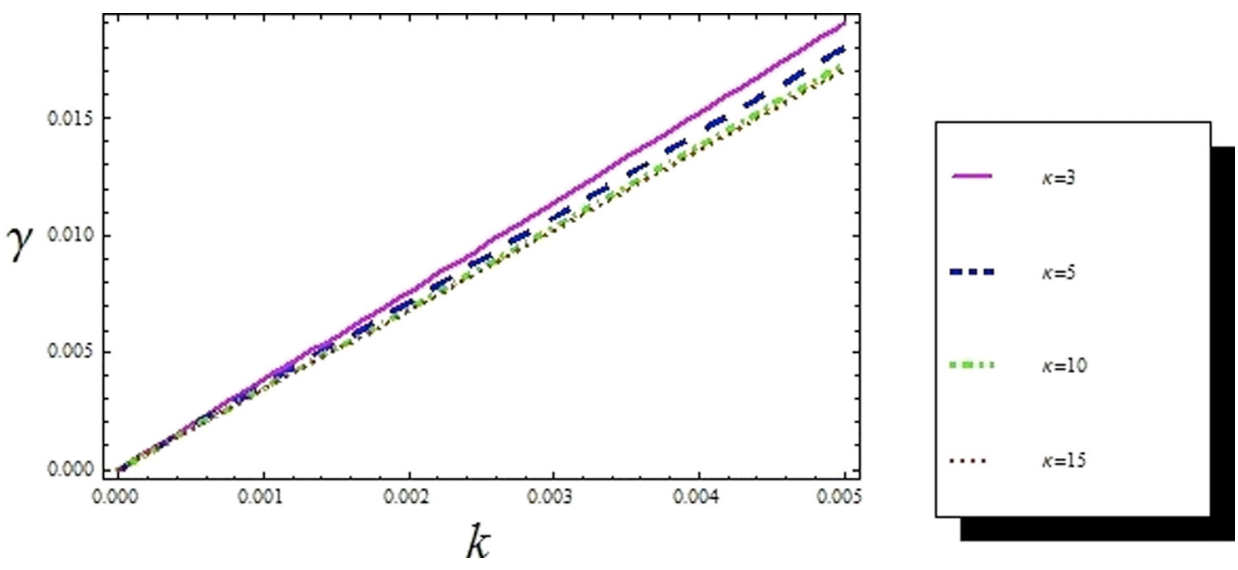

FIG. 1. The growth rate $\gamma$ is plotted against the wave number $k$ such that $v_{\phi} \gg \theta_{\|_{\text {oi }}}$ for the different values of kappa, i.e., $\kappa=3$ (purple solid line), $\kappa=5$ (blue dashed line), $\quad \kappa=10$ (green dotted-dashed line), and $\kappa=15$ (red dotted line) for fixed value of $v_{0}=8 \times 10^{7}$. 


$$
\begin{aligned}
& \gamma=\sqrt{\frac{\pi}{8}} \frac{\Gamma(\kappa+1)}{\left(\kappa-\frac{3}{2}\right)^{3 / 2} \Gamma\left(\kappa-\frac{1}{2}\right)} \frac{k \omega_{p_{d}} \lambda_{D_{2}}^{3}}{\left(\frac{\left(\kappa-\frac{1}{2}\right)}{\left(\kappa-\frac{3}{2}\right)}+k^{2} \lambda_{D_{2}}^{2}\right)^{3 / 2}} \\
& \left.\times\left\{\frac{v_{0}}{\omega_{p_{w e}} \lambda_{D_{w e}}^{3}}+\frac{v_{0}}{\omega_{p_{w i}} \lambda_{D_{w i}}^{3}}\left[1+\left(1-\frac{k v_{0}}{\omega}\right)^{2}\left(\frac{v_{T_{\|_{d}}}^{2} / v_{T_{\|_{w i}}}^{2}}{(2 \kappa-3)\left(2 \frac{\left(\kappa-\frac{1}{2}\right)}{\left(\kappa-\frac{3}{2}\right)}+k^{2} \lambda_{D_{2}}^{2}\right)}\right)^{\lambda_{D_{d}}^{2}}+\frac{3 v_{T_{\|_{d}}}^{2} / v_{T_{\|_{w i}}}^{2}}{(2 \kappa-3)}\right)^{-\kappa-1}\right)^{2}\right] \\
& -\frac{v_{T_{\|_{d}}}}{\sqrt{\left(2 \frac{\left(\kappa-\frac{1}{2}\right)}{\left(\kappa-\frac{3}{2}\right)}+k^{2} \lambda_{D_{2}}^{2}\right)}} \frac{\lambda_{D_{2}}}{\lambda_{D_{d}}}\left[\frac{1}{\omega_{p_{c i}} \lambda_{D_{c i}}^{3}}+\frac{1}{\omega_{p_{c e}} \lambda_{D_{c e}}^{3}}+\frac{1}{\omega_{p_{w e}} \lambda_{D_{w e}}^{3}}+\frac{1}{\omega_{p_{w i}} \lambda_{D_{w i}}^{3}}\right. \\
& \times\left[1+\left(1-\frac{k v_{0}}{\omega}\right)^{2}\left(\frac{v_{T_{\|_{d}}}^{2} / v_{T_{\|_{w i}}}^{2}}{(2 \kappa-3)\left(2 \frac{\left(\kappa-\frac{1}{2}\right)}{\left(\kappa-\frac{3}{2}\right)}+k^{2} \lambda_{D_{2}}^{2}\right)}\left(\frac{\lambda_{D_{2}}^{2}}{\lambda_{D_{d}}^{2}}\right)+\frac{3 v_{T_{\|_{d}}}^{2} / v_{T_{\|_{w i}}}^{2}}{(2 \kappa-3)}\right]\right]^{-\kappa-1} \\
& \left.\left.+\frac{1}{\omega_{p_{d}} \lambda_{D_{d}}^{3}}\left[1+\frac{1}{(2 \kappa-3)\left(2 \frac{\left(\kappa-\frac{1}{2}\right)}{\left(\kappa-\frac{3}{2}\right)}+k^{2} \lambda_{D_{2}}^{2}\right)}\left(\frac{\lambda_{D_{2}}^{2}}{\lambda_{D_{d}}^{2}}\right)+\frac{3}{(2 \kappa-3)}\right]^{-\kappa-1}\right]\right\} \text {. }
\end{aligned}
$$

This equation can also be expressed in the non-dimensional form as
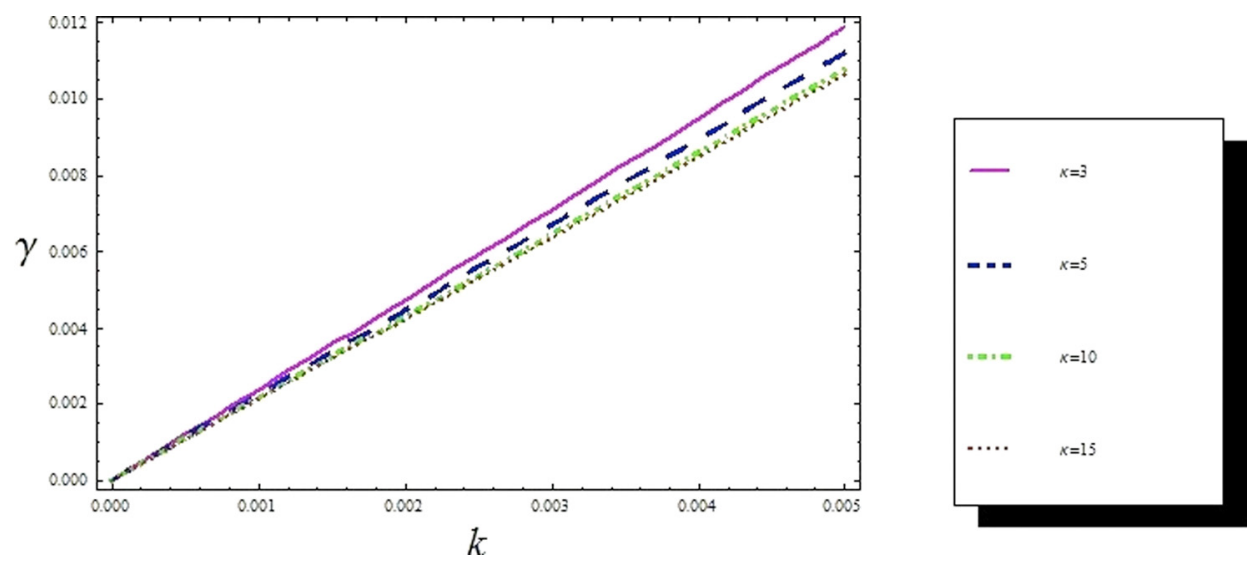

FIG. 2. The growth rate $\gamma$ is plotted against the wave number $k$ such that $v_{\phi} \gg \theta_{\|_{\omega i}}$ for the different values of kappa, i.e., $\kappa=3$ (purple solid line), $\kappa=5$ (blue dashed line), $\kappa=10$ (green dotted-dashed line), and $\kappa=15$ (red dotted line) for fixed value of $v_{0}=5 \times 10^{7}$. 


$$
\begin{aligned}
& \frac{\gamma}{\omega_{p_{d}}}=\sqrt{\frac{\pi}{8}} \frac{\Gamma(\kappa+1)}{\left(\kappa-\frac{3}{2}\right)^{3 / 2} \Gamma\left(\kappa-\frac{1}{2}\right)} \frac{k \lambda_{D_{d}} \omega_{p_{d}} \lambda_{D_{2}}^{3}}{\left(\frac{\left(\kappa-\frac{1}{2}\right)}{\left(\kappa-\frac{3}{2}\right)}+k^{2} \lambda_{D_{2}}^{2}\right)^{3 / 2}}\left\{\frac{v_{0} / v_{T_{\|_{d}}}}{\omega_{p_{w e}} \lambda_{D_{w e}}^{3}}+\frac{v_{0} / v_{T_{\|_{d}}}}{\omega_{p_{w i}} \lambda_{D_{w i}}^{3}}\right.
\end{aligned}
$$

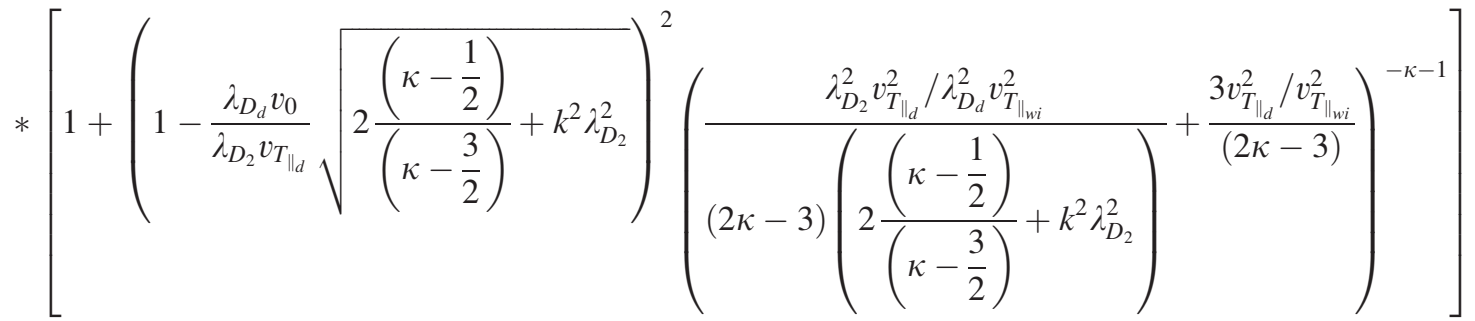

$$
\begin{aligned}
& -\frac{1}{\sqrt{\left(2 \frac{\left(\kappa-\frac{1}{2}\right)}{\left(\kappa-\frac{3}{2}\right)}+k^{2} \lambda_{D_{2}}^{2}\right)}} \frac{\lambda_{D_{2}}}{\lambda_{D_{d}}}\left[\frac{1}{\omega_{p_{c i}} \lambda_{D_{c i}}^{3}}+\frac{1}{\omega_{p_{c e}} \lambda_{D_{c e}}^{3}}+\frac{1}{\omega_{p_{w e}} \lambda_{D_{w e}}^{3}}+\frac{1}{\omega_{p_{w i}} \lambda_{D_{w i}}^{3}}\right.
\end{aligned}
$$

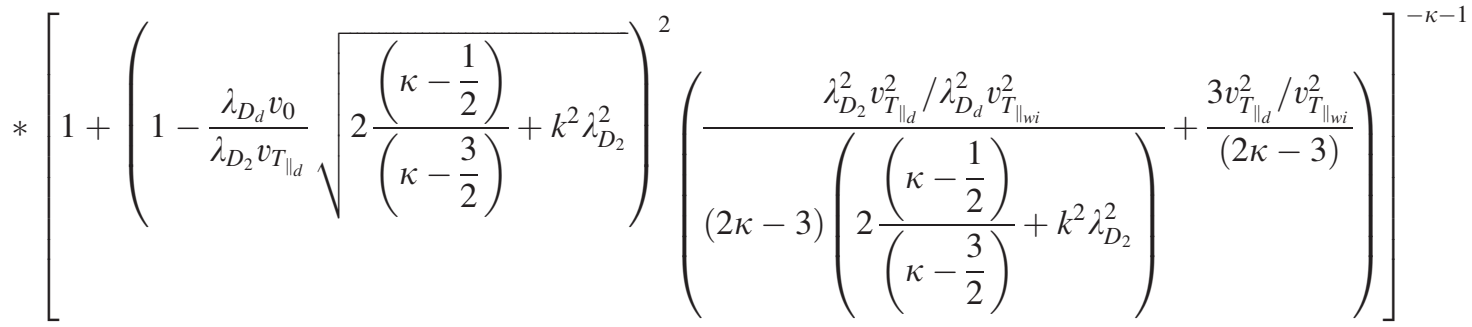

$$
\begin{aligned}
& \left.\left.+\frac{1}{\omega_{p_{d}} \lambda_{D_{d}}^{3}}\left[1+\frac{1}{(2 \kappa-3)\left(2 \frac{\left(\kappa-\frac{1}{2}\right)}{\left(\kappa-\frac{3}{2}\right)}+k^{2} \lambda_{D_{2}}^{2}\right)}\left(\frac{\lambda_{D_{2}}^{2}}{\lambda_{D_{d}}^{2}}\right)+\frac{3}{(2 \kappa-3)}\right]^{-\kappa-1}\right]\right\} \text {. }
\end{aligned}
$$

As $\kappa \rightarrow \infty$, our equation reduces to the Maxwellian case, having the following form:

$$
\frac{\gamma}{\omega_{p_{d}}}=\sqrt{\frac{\pi}{8}} \frac{k \lambda_{D_{d}} \omega_{p_{d}} \lambda_{D_{2}}^{3}}{\left(2+k^{2} \lambda_{D_{2}}^{2}\right)^{3 / 2}}\left\{\frac{v_{0} / v_{T_{\|_{d}}}}{\omega_{p_{w e}} \lambda_{D_{w e}}^{3}}+\frac{v_{0} / v_{T_{\|_{d}}}}{\omega_{p_{w i}} \lambda_{D_{w i}}^{3}}-\frac{1}{\sqrt{\left(2+k^{2} \lambda_{D_{2}}^{2}\right)}} \frac{\lambda_{D_{2}}}{\lambda_{D_{d}}}\left[\frac{1}{\omega_{p_{c i}} \lambda_{D_{c i}}^{3}}+\frac{1}{\omega_{p_{c e}} \lambda_{D_{c e}}^{3}}+\frac{1}{\omega_{p_{w e}} \lambda_{D_{w e}}^{3}}+\frac{1}{\omega_{p_{w i}} \lambda_{D_{w i}}^{3}}\right]\right\} .
$$

For small phase velocity limit of solar wind ions, $v_{\phi} \ll \theta_{\|_{\omega i}}$, our dielectric response function is modified resulting in
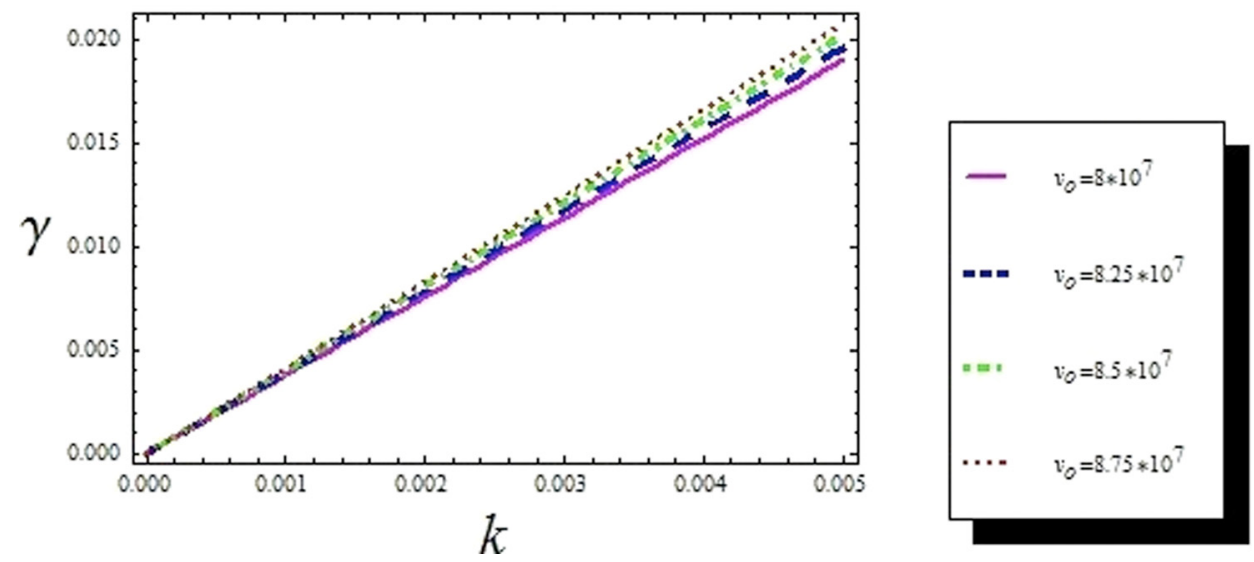

FIG. 3. The growth rate $\gamma$ is plotted against the wave number $k$ such that $v_{\phi} \gg \theta_{\|_{\omega i}}$ for the different values of streaming velocities, i.e., $v_{0}=8 \times 10^{7}$ (purple solid line), $v_{0}=8.25 \times 10^{7}$ (blue dashed line), $v_{0}=8.5 \times 10^{7}$ (green dotted-dashed line), and $v_{0}=8.75 \times 10^{7}$ (red dotted line) for the fixed value of $\kappa=3$. 


$$
\begin{aligned}
\epsilon^{\prime}(k, \omega)= & \left.\left(2 \frac{\left(\kappa-\frac{1}{2}\right)}{\left(\kappa-\frac{3}{2}\right)}+k^{2} \lambda_{D_{1}}^{2}\right)-\frac{k^{2} \lambda_{D_{1}}^{2} \omega_{p_{d}}^{2}}{\omega^{2}}\left[1+\frac{k^{2} \theta_{\|_{d}}^{2}}{\omega^{2}} \frac{3 \kappa}{2\left(\kappa-\frac{3}{2}\right)}\right]\right] \\
& +2 i \sqrt{\pi} \frac{\lambda_{D_{1}}^{2} \Gamma(\kappa+1)}{\kappa^{\frac{3}{2}} \Gamma\left(\kappa-\frac{1}{2}\right)}\left[\frac{\omega / k}{\omega_{p_{c i}} \lambda_{D_{c i}}^{3}}+\frac{\omega / k}{\omega_{p_{c e}} \lambda_{D_{c e}}^{3}}+\frac{\omega / k-v_{0}}{\omega_{p_{\omega e}} \lambda_{D_{\omega e}}^{3}}+\frac{\omega / k-v_{0}}{\omega_{p_{\omega i}} \lambda_{D_{\omega i}}^{3}}+\frac{\omega / k}{\omega_{p_{d}} \lambda_{D_{d}}^{3}}\left[1+\frac{\left(\frac{\omega}{k}\right)^{2}}{\kappa \theta_{\|_{d}}^{2}}\right]^{-\kappa-1}\right],
\end{aligned}
$$

where $\lambda_{D_{1}}^{-2}=\lambda_{D_{c i}}^{-2}+\lambda_{D_{\omega e}}^{-2}+\lambda_{D_{c e}}^{-2}+\lambda_{D_{\omega i}}^{-2}$. This also modifies the real $\omega$ leading to

$$
\omega_{r}^{2}=\frac{T_{c e} / m_{d}}{\left(1+\frac{T_{c e}}{T_{c i}} \frac{n_{c i 0}}{n_{c e 0}}+\frac{T_{c e}}{T_{w e}} \frac{n_{w e 0}}{n_{c e 0}}+\frac{T_{c e}}{T_{w i}} \frac{n_{w i 0}}{n_{c e 0}}\right)} \frac{k^{2} Z_{d}^{2} n_{d o} / n_{c e 0}}{\left(\kappa-\frac{1}{2}\right)}\left(1+\frac{k^{2} \theta_{\|_{d}}^{2}}{\omega^{2}} \frac{3 \kappa}{2\left(\kappa-\frac{3}{2}\right)}\right] .
$$

Similarly, the modified growth rate $\gamma^{\prime}$ becomes

$$
\gamma^{\prime}=Y\left[v_{0} b-\frac{\omega_{r}}{k}(a+b+c)\right],
$$

where

and

$$
b=\frac{1}{\omega_{p_{w e}} \lambda_{D_{w e}}^{3}}+\frac{1}{\omega_{p_{w i}} \lambda_{D_{w i}}^{3}},
$$

$$
c=\frac{1}{\omega_{p_{d}} \lambda_{D_{d}}^{3}}\left[1+\frac{1}{(2 \kappa-3)\left(2 \frac{\left(\kappa-\frac{1}{2}\right)}{\left(\kappa-\frac{3}{2}\right)}+k^{2} \lambda_{D 1}^{2}\right)} \frac{\lambda_{D_{1}}^{2}}{\lambda_{D_{d}}^{2}}+\frac{3}{(2 \kappa-3)} \frac{\lambda_{D_{d}}^{2}}{\lambda_{D_{1}}^{2}}\right]^{-\kappa-1},
$$

So, the condition for the instability now comes out to be

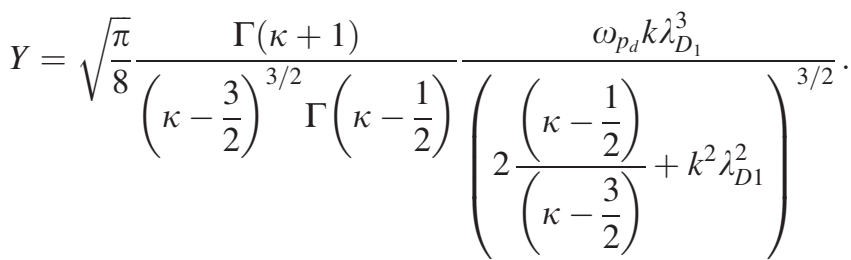

$$
v_{0}>\frac{\omega_{r}}{k}\left(1+\frac{a+c}{b}\right) .
$$

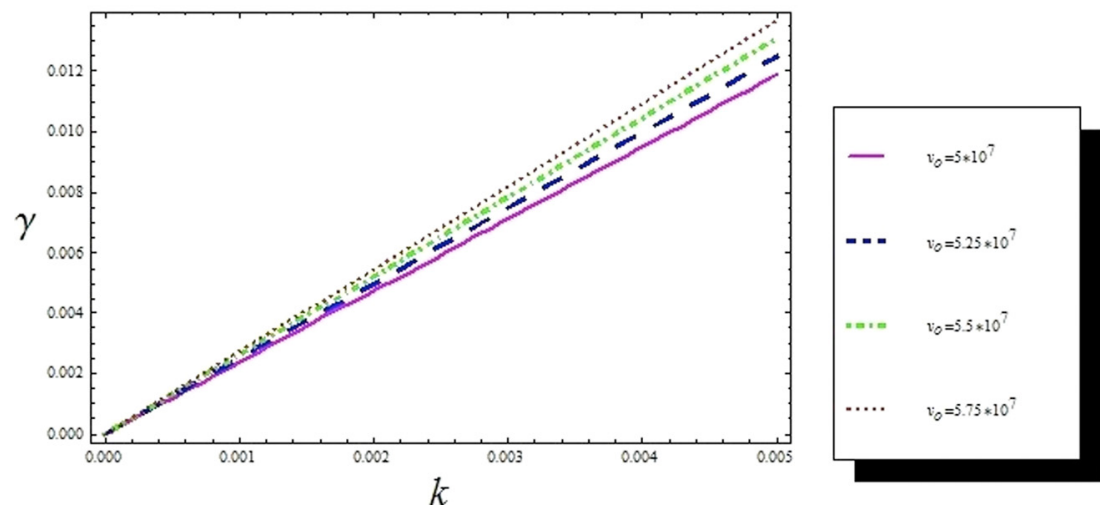

FIG. 4. The growth rate $\gamma$ is plotted against the wave number $k$ such that $v_{\phi} \gg \theta_{\|_{\omega i}}$ for the different values of streaming velocities, i.e., $v_{0}=5 \times 10^{7}$ (purple solid line), $v_{0}=5.25 \times 10^{7}$ (blue dashed line), $v_{0}=5.5 \times 10^{7}$ (green dotted-dashed line), and $v_{0}=5.75 \times 10^{7}$ (red dotted line) for the fixed value of $\kappa=3$. 
With substitution of $\omega_{r}$, we get (normalized) growth rate given by

$$
\begin{aligned}
\frac{\gamma^{\prime}}{\omega_{p_{d}}}= & \sqrt{\frac{\pi}{8}} \frac{\Gamma(\kappa+1)}{\left(\kappa-\frac{3}{2}\right)^{3 / 2} \Gamma\left(\kappa-\frac{1}{2}\right)} \overline{\left(2 \frac{\left(\kappa-\frac{1}{2}\right)}{\left(\kappa-\frac{3}{2}\right)}+k^{2} \lambda_{D 1}^{2}\right)^{3 / 2}} \omega_{p_{d}} \lambda_{D_{1}}^{3}\left\{\frac{v_{0}}{v_{T_{\|_{d}}}}\left(\frac{1}{\omega_{p_{w e}} \lambda_{D_{w e}}^{3}}+\frac{1}{\omega_{p_{w i}} \lambda_{D_{w i}}^{3}}\right)\right. \\
& -\frac{\lambda_{D_{1}}}{\sqrt{\left(2 \frac{\left(\kappa-\frac{1}{2}\right)}{\left(\kappa-\frac{3}{2}\right)}+k^{2} \lambda_{D_{2}}^{2}\right)}} \lambda_{D_{d}}\left[\frac{1}{\omega_{p_{c i}} \lambda_{D_{c i}}^{3}}+\frac{1}{\omega_{p_{c e}} \lambda_{D_{c e}}^{3}}+\frac{1}{\omega_{p_{w e}} \lambda_{D_{w e}}^{3}}+\frac{1}{\omega_{p_{w i}} \lambda_{D_{w i}}^{3}}+\frac{1}{\omega_{p_{d}} \lambda_{D_{d}}^{3}}\right.
\end{aligned}
$$

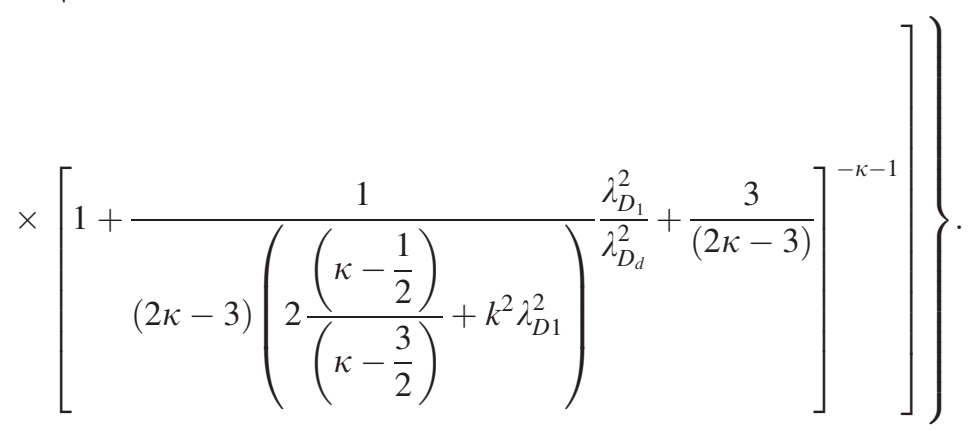

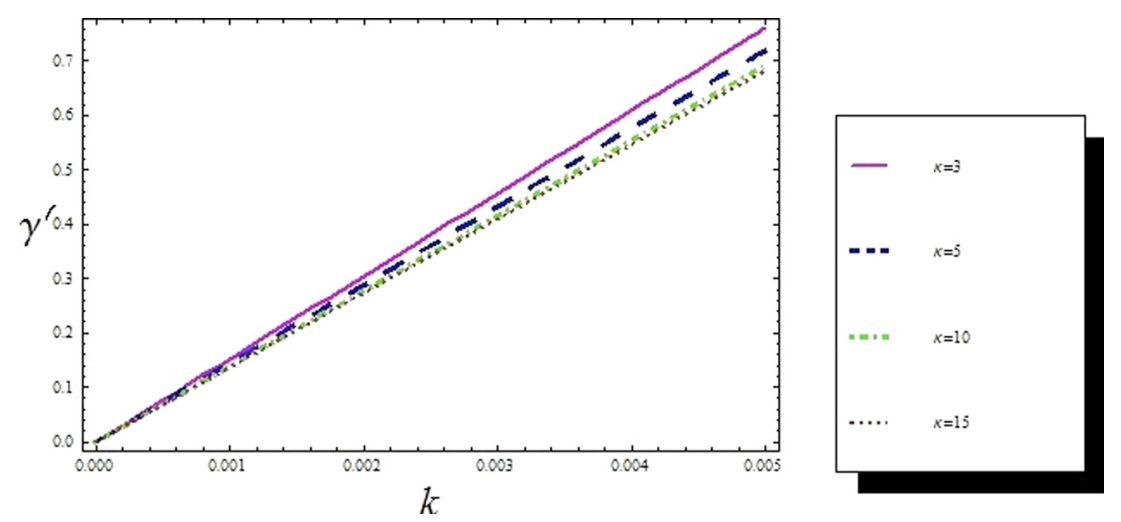

FIG. 5. The growth rate $\gamma$ is plotted against the wave number $k$ such that $v_{\phi} \ll \theta_{\|_{\omega i}}$ for the different values of kappa, i.e., $\kappa=3$ (purple solid line), $\kappa=5$ (blue dashed line), $\kappa=10$ (green dotted-dashed line), and $\kappa=15$ (red dotted line) for fixed value of $v_{0}=8 \times 10^{7}$.
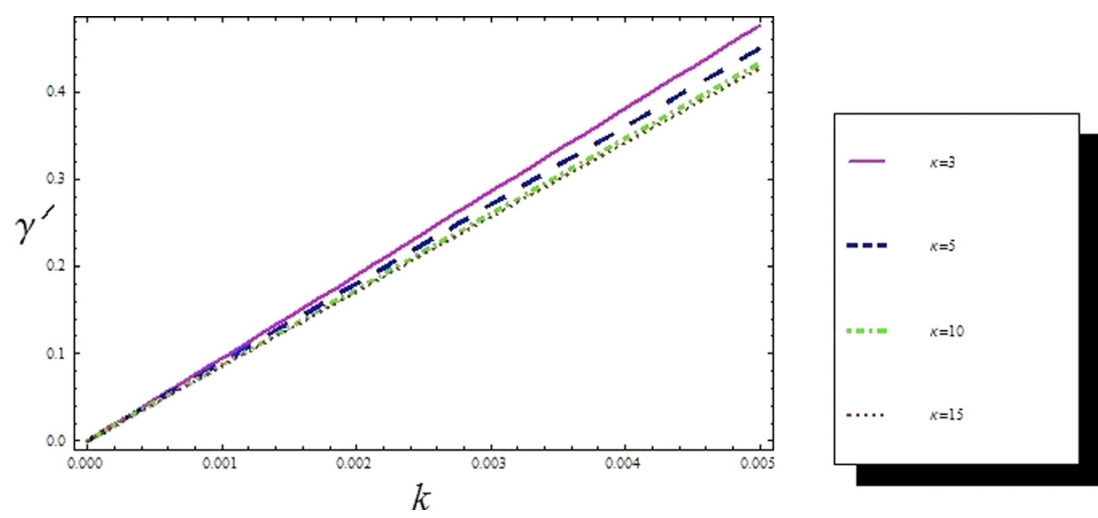

FIG. 6. The growth rate $\gamma$ is plotted against the wave number $k$ such that $v_{\phi} \ll \theta_{\|_{\omega i}}$ for the different values of kappa, i.e., $\kappa=3$ (purple solid line), $\kappa=5$ (blue dashed line), $\quad \kappa=10$ (green dotted-dashed line), and $\kappa=15$ (red dotted line) for fixed value of $v_{0}=5 \times 10^{7}$. 
Again in the limit $\kappa \rightarrow \infty$, all the Maxwellian counterparts of the growth rate should be

$$
\begin{aligned}
& \frac{\gamma^{\prime}}{\omega_{p_{d}}}=\sqrt{\frac{\pi}{8}} \frac{k \lambda_{D_{d}} \omega_{p_{d}} \lambda_{D_{1}}^{3}}{\left(2+k^{2} \lambda_{D 1}^{2}\right)^{3 / 2}}\left\{\frac{v_{0}}{v_{T_{\|_{d}}}}\left(\frac{1}{\omega_{p_{w e}} \lambda_{D_{w e}}^{3}}+\frac{1}{\omega_{p_{w i}} \lambda_{D_{w i}}^{3}}\right)\right.
\end{aligned}
$$

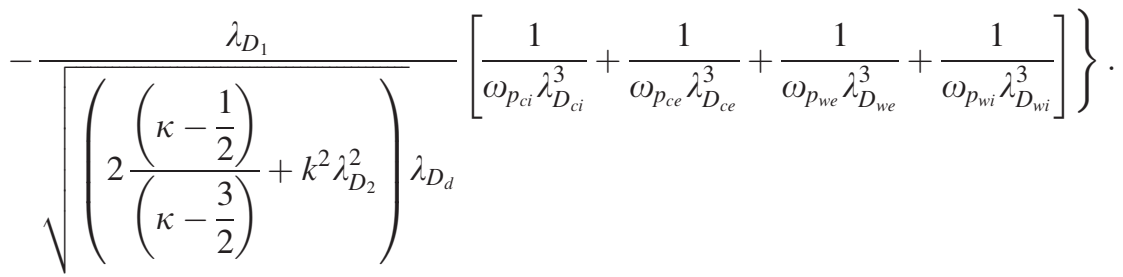

\section{QUANTITATIVE ANALYSIS AND CONCLUSIONS}

To validate the results found in this letter, we make use of the typical parameters relevant to the observed situations. ${ }^{1,2}$ Particularly, we analyze the impact of the spectral index and streaming velocity on the instability growth of the nonMaxwellian permeating dusty plasma mode. In Figs. 1 and 2, the growth rate $\gamma$ is plotted against the wave number $k$ for different values of spectral index $\kappa$ with the variation of streaming velocities, i.e., $v_{0}=8 \times 10^{7} \mathrm{~cm} / \mathrm{s}$ and $v_{0}=5 \times 10^{7} \mathrm{~cm} / \mathrm{s}$, respectively, in the large phase velocity limit of solar wind ions. It can be clearly seen from the figure that growth rates of the wave are increased with the decrease in spectral index. Physically, it points to the presence of some source of energy which excites the wave and is dominant at smaller value of spectral index. That is why the growth of the wave is large at $\kappa=3$ as compared with $\kappa=15$. Figs. 3 and 4 are presenting the effect of streaming velocity $v_{0}$ on the growth rate $\gamma$ of the acoustic wave in the large phase velocity regime for the solar wind ions. It is evident from the plots that increase in the streaming velocity $v_{0}$ increases the growth rate $\gamma$ of the acoustic wave. This shows the increase in the streaming velocity with the free energy strength. The last four figures are drawn in the limit of small phase velocity for solar wind ions and showing the effect of spectral index kappa and streaming velocity on the modified growth rate $\gamma^{\prime}$. Figs. 5 and 6 are showing an increase in the growth rate $\gamma^{\prime}$ with the decrease in the value of spectral index $\kappa$ but here the phase velocity of solar wind ions is smaller than their thermal velocities. Due to small phase velocity of ions in the solar wind plasma, the superthermal particles imparting energy to the wave increases and wave exhibits large excitation in the fifth and sixth figure
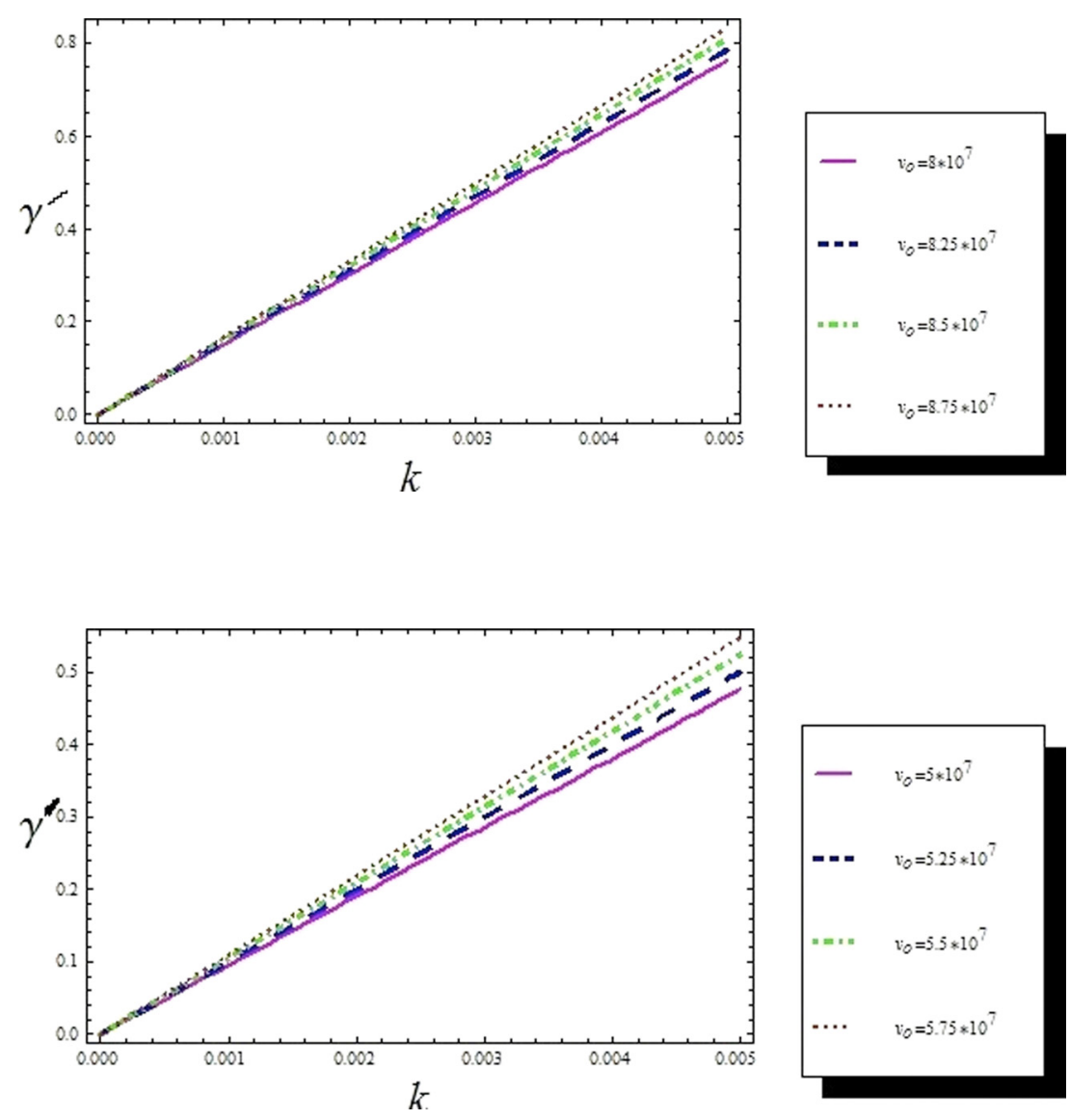

FIG. 7. The growth rate $\gamma$ is plotted against the wave number $k$ such that $v_{\phi} \ll \theta_{\|_{\omega i}}$ for the different values of streaming velocities, i.e., $v_{0}=8 \times 10^{7}$ (purple solid line), $v_{0}=8.25 \times 10^{7}$ (blue dashed line), $v_{0}=8.5 \times 10^{7}$ (green dotted-dashed line), and $v_{0}=8.75 \times 10^{7}$ (red dotted line) for the fixed value of $\kappa=3$.

FIG. 8. The growth rate $\gamma$ is plotted against the wave number $k$ such that $v_{\phi} \ll \theta_{\|_{\omega i}}$ for the different values of streaming velocities, i.e., $v_{0}=5 \times 10^{7}$ (purple solid line), $v_{0}=5.25 \times 10^{7}$ (blue dashed line), $v_{0}=5.5 \times 10^{7}$ (green dotted-dashed line), and $v_{0}=5.75 \times 10^{7}$ (red dotted line) for the fixed value of $\kappa=3$. 
in comparison with first two figures. Finally, Figs. 7 and 8 are showing increase in the growth rates $\gamma^{\prime}$ with the enhancement in the streaming velocity $v_{0}$. The significant difference shown is the large growth rate $\gamma^{\prime}$ in comparison with $\gamma$ as expected.

Conclusively, we have investigated the dispersive properties and a kinetic instability associated with a low frequency (dust-acoustic) mode in a non-Maxwellian permeating plasma modelled by a generalized Lorentzian (kappa) distribution function. The threshold for the instability is found under the various limits of the wave phase velocity. Such type of dynamics is of interest when the solar wind is streaming through the cometary plasma in the presence of interstellar dust. In the limiting cases of phase velocity of the waves larger and smaller than the thermal velocity of dust particles, the analytical and graphical studies of the dispersion and growth rate of dust-acoustic mode are shown. The streaming of solar wind disturbs the stability of the plasma system and plasma becomes unstable. This instability leads to a growth in the wave spectrum of the plasma which is the matter of interest in our present studies and can be interesting for readers in the area.

\section{ACKNOWLEDGMENTS}

We thank the anonymous referee for useful suggestions to improve the manuscript. One of us (Z.E.) acknowledges Professor Nodar Tsintsadze, Ciro Marmolino and Jovo Vranjes for the fruitful discussions and the financial contribution of the Grant Ref. No. CUO H31J11000060005 between Universia' del Molise - and Consorzio di Ricerca per l'Energia e le Applicazioni Tecnologiche dell'Elettromagnetismo(CREATE)-ItalyResearch Consortium for Energy and Technological Applications of Electromagnetism.
${ }^{1}$ J. Vranjes, S. Poedts, and Z. Ehsan, Phys. Plasmas 16, 074501 (2009).

${ }^{2}$ J. Vranjes, Phys. Plasmas 18, 084501 (2011).

${ }^{3}$ J. Vranjes, Astron. Astrophys. 554, A90 (2013).

${ }^{4}$ Z. Liu and J. Du, Phys. Plasmas 16, 123707 (2009).

${ }^{5}$ J. Gong, Z. Liu, and J. Du, Phys Plasmas 19, 043704 (2012) and references therein.

${ }^{6}$ D. A. Mendis, Plasma Sources Sci. Technol. 11, A219 (2002).

${ }^{7}$ Z. Ehsan, N. L. Tsintsadze, and S. Poedts, AIP Conf. Proc. 1397, 383 (2011); N. L. Tsintsadze, G. Murtaza, and Z. Ehsan, Phys. Plasmas 13, 22103 (2006).

${ }^{8}$ S. Ghosh, Z. Ehsan, and G. Murtaza Phys. Plasmas 15, 023701 (2008).

${ }^{9}$ I. Alexeff, R. V. Neidigh, W. F. Peeds, and E. D. Shiply, Phys. Rev. Lett. 10, 273 (1963).

${ }^{10}$ V. M. Vasyliunas, J. Geophys. Res. 73, 2839, doi:10.1029/JA073i009p02839 (1968).

${ }^{11}$ S. Olbert, in Physics of the Magnetosphere, edited by R. L. Carovillano, J.

F. McClay, and H. R. Radoski (Reidel, Dordrecht, Holland, 1969), p. 641.

${ }^{12}$ D. Summers and R. M. Thorne, Phys. Fluids B 3, 1835 (1991).

${ }^{13}$ M. A. Hellberg and R. L. Mace, Phys. Plasmas 9, 1495 (2002).

${ }^{14}$ L. Y. Liu and J. L. Du, Physica A 387, 4821 (2008).

${ }^{15}$ Z. P. Liu, L. Y. Liu, and J. L. Du, Phys. Plasmas 16, 072111 (2009).

${ }^{16}$ S. Ali Shan and A. Mushtaq, Astrophys. Space. Sci. 346, 367 (2013); S. Ali Shan, S. A. El-Tantawy, and W. M. Moslem Phys. Plasmas 20, 082104 (2013).

${ }^{17}$ J. Gong, Z. Liu, and J. Du Phys. Plasmas 19, 083706 (2012).

${ }^{18}$ J. L. Du and Y. L. Song, "Solar wind speed theory and the nonextensivity of solar corona," in Proceedings of the Third UN/ESA/NASA Workshop on the International Heliophysical Year 2007 and Basic Space Science, Astrophysics and Space Science Proceedings, edited by H. J. Haubold and A. M. Mathai (Springer, Berlin, 2010), p. 93102.

${ }^{19}$ M. P. Leubner and Z. Voros, Astrophys. J. 618, 547 (2005).

${ }^{20}$ D. Jiulina, Phys. Plasmas 20, 092901 (2013).

${ }^{21}$ G. Livadiotis and D. J. McComas, Space Sci. Rev. 175, 183 (2013).

${ }^{22}$ M. P. Leubner and N. Schupfer, Nonlinear Process. Geophys. 9, 75 (2002).

${ }^{23}$ R. A. Treumann, Phys. Scr. 59, 19 (1999).

${ }^{24}$ R. Sabry, W. M. Moslem, and P. K. Shukla, Phys. Plasmas 16, 032302 (2009).

${ }^{25}$ K. Arshad and S. Mahmood, Phys. Plasmas 17, 124501 (2010).

${ }^{26}$ K. Arshad, S. Mahmood, and A. M. Mirza, Phys. Plasmas 18, 092115 (2011).

${ }^{27}$ S. Sultana, I. Kourakis, N. S. Saini, and M A. Hellberg, Phys. Plasmas 17, 032310 (2010). 